\title{
ESPECTROSCOPIA DE FOTOELÉTRONS DE LIMIARES DE ÁTOMOS E MOLÉCULAS
}

\author{
Maria Cristina Andreolli Lopes*, Helder Couto, Cleber Dias Moreira e Helen Silva \\ Departamento de Física, Instituto de Ciências Exatas, Universidade Federal de Juiz de Fora, 36036-330 Juiz de Fora - MG
}

Recebido em 12/11/04; aceito em 27/4/05; publicado na web em 24/8/05

\begin{abstract}
ATOMIC AND MOLECULAR THRESHOLD PHOTOELECTRON SPECTROSCOPY. A threshold photoelectron spectrometer applied to the study of atomic and molecular threshold photoionization processes is described. The spectrometer has been used in conjunction with a toroidal grating monochromator at the National Synchrotron Radiation Laboratory (LNLS), Brazil. It can be tuned to accept threshold electrons $(<20 \mathrm{meV})$ and work with a power resolution of $716(\sim 18 \mathrm{meV}$ at $12 \mathrm{eV})$ with a high signal/ noise ratio. The performance of this apparatus and some characteristics of the TGM (Toroidal Grating Monochromator) beam line of LNLS are described and discussed by means of argon, $\mathrm{O}_{2}$ and $\mathrm{N}_{2}$ threshold photoelectron spectra.
\end{abstract}

Keywords: threshold photoelectron spectroscopy; synchrotron radiation; photoionization.

\section{INTRODUÇÃO}

A Espectroscopia de Fotoeléctroms de Limiares ("TPES Threshold Photoelectron Spectroscopy") ${ }^{1-4}$ tem se mostrado uma importante ferramenta no estudo de estados eletrônicos de íons atômicos e moleculares. Através dela pode-se obter, além das informações geralmente acessíveis pela Espectroscopia de Fotoelétrons convencional ("PES - Photoelectron Spectroscopy"), dados sobre estados populados por rotas indiretas de excitação, envolvendo estados neutros altamente excitados que decaem por autoionização para um estado iônico degenerado próximo, produzindo um elétron de baixa energia.

A TPES distingue-se por apresentar, simultaneamente, características de alta sensibilidade e resolução em energia e, também, por ser livre de alargamento Doppler. Estas características possibilitam a aquisição de espectros ricos em estruturas, registrando processos com baixa seção de choque.

Na TPES a energia dos fótons é variada continuamente, enquanto o analisador é sintonizado para detectar fotoelétrons com energia nominalmente zero. À medida que a energia dos fótons cruza os limiares de ionização, são ejetados elétrons com energias muito próximas de zero, mapeando diretamente os níveis de energia dos estados iônicos produzidos por rotas diretas e indiretas, como esquematizado na Figura 1 . Na fotoionização direta, um fóton é absorvido pelo alvo, resultando na sua pronta ionização, com a ejeção de um elétron com energia residual próxima a zero. Já na indireta, o alvo é levado a um estado superexcitado (estado de Rydberg) localizado alguns meV acima de um excitado iônico mais baixo, decaindo para este estado iônico por autoionização. Esta técnica pode ser empregada tanto no estudo da camada de valência como também, de camada interna ${ }^{5,6}$. A fotoionização de moléculas logo acima do limiar geralmente fornece uma nova perspectiva ao processo de ionização. Novos mecanismos são induzidos, onde a correlação eletrônica tem um papel importante. Por ex., os espectros da camada de valência interna e também da camada interna de átomos e moléculas são ricamente estruturados por estados satélites, que correspondem a estados excitados de íons, formados devido às correlações eletrônicas ${ }^{5,7}$.

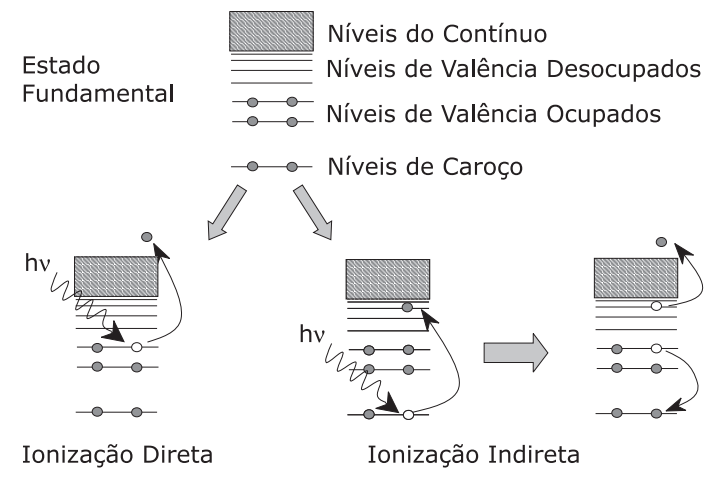

Figura 1: Diagrama de níveis de energia de um átomo em uma molécula. $\mathrm{Na}$ PES observam-se somente os processos diretos de ionização. Já na TPES, como a energia dos fótons é variada continuamente, além dos processos diretos, são também registrados os processos indiretos de ionização, envolvendo a autoionização do alvo

Com o objetivo de elucidar mecanismos de relaxação de estados excitados localizados próximos aos limiares de ionização de moléculas poliatômicas, construiu-se um Espectrômetro de Fotoelétrons de Limiares $^{8}$. A TPES utiliza a Técnica de Campo Penetrante proposta em 1974 por Cvejanovic e Read ${ }^{9}$, originalmente usada no estudo de átomos e moléculas por impacto de elétrons. Posteriormente King e colaboradores ${ }^{10}$ aperfeiçoaram a Técnica de Campo Penetrante e a adaptaram para o impacto de fótons, obtendo altíssima eficiência na detecção de elétrons com energias menores ou iguais a $3 \mathrm{meV}$. A partir destes estudos pioneiros, a Técnica de Campo Penetrante associada à radiação síncrotrônica, isto é, a TPES vem sendo aplicada essencialmente no estudo gases nobres e moléculas pequenas. Neste trabalho apresentamos as características do espectrômetro TPE construído em nosso laboratório, bem como seu desempenho associado à linha de luz TGM ("Toroidal Grating Monochromator") do Laboratório Nacional de Luz Síncrotron (LNLS), que cobre a região de energia de 12 a $300 \mathrm{eV}$.

\section{PARTE EXPERIMENTAL}

O espectrômetro de Fotoelétrons de Limiares consiste basica- 
mente da fonte de luz sintonizável UV, uma fonte de gás efusivo e um analisador de energia de elétrons, como mostra a Figura 2.

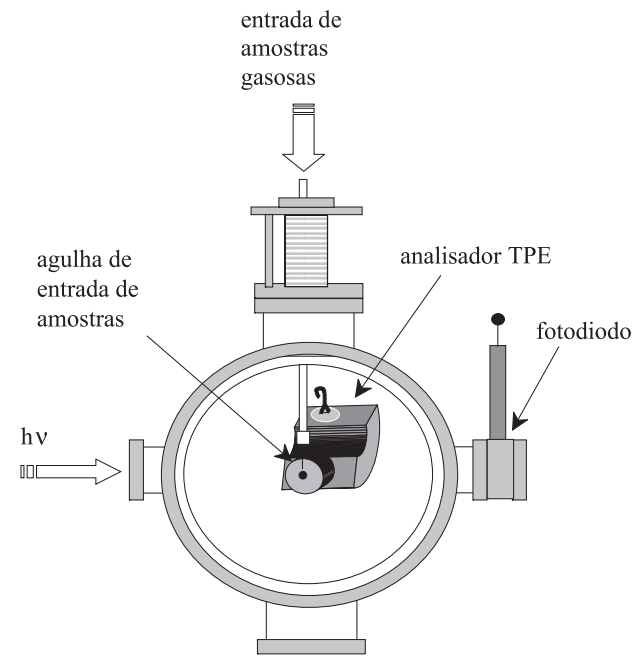

Figura 2. Arranjo experimental empregado na Espectroscopia de Fotoelétrons de Limiares

O feixe de fótons monocromatizado proveniente da linha TGM, é entregue na região de colisão através de um capilar de Pyrex, com diâmetro interno de $2 \mathrm{~mm}$. A extremidade do capilar é aterrada, a fim de prevenir efeitos de carga eletrostática que afetariam a eficiência de extração dos elétrons com energias próximas a zero, que são ejetados na ionização do alvo. A intensidade da fonte de luz é monitorada durante a aquisição dos espectros através de um fotodiodo de $\mathrm{Al}_{2} \mathrm{O}_{3}$ posicionado no eixo do feixe de fótons, aproximadamente a $25 \mathrm{~cm}$ da região de interação, para normalização do sinal registrado pelo detector. O feixe gasoso é formado por efusão, usando uma agulha com diâmetro interno de $0,2 \mathrm{~mm}$, situada aproximadamente $3 \mathrm{~mm}$ acima do feixe de fótons. Aplica-se um potencial na agulha a fim de melhorar a eficiência de detecção dos elétrons de baixa energia. A câmara de alto vácuo que aloja o analisador é conectada à linha de luz através de um tubo flexível e mantida uma pressão residual típica de $5 \times 10^{-7}$ mbar. A câmara é aquecida durante todo o experimento, ficando a uma temperatura constante de aproximadamente $70{ }^{\circ} \mathrm{C}$, para manter maior estabilidade do espectrômetro após a introdução das amostras gasosas. Esta câmara é internamente revestida com uma camada de aproximadamente $0,05 \mathrm{~cm}$ de mu-metal, o que reduz os campos magnéticos residuais na região de colisão para valores inferiores a 2 mGauss, evitando distorções nas trajetórias dos elétrons ejetados. $\mathrm{O}$ analisador de elétrons foi construído em nosso laboratório, segundo a proposta de King e colaboradores ${ }^{10}$ que aplica a Técnica de Campo Penetrante 9 . Nesta técnica, produz-se na região de colisão um campo eletrostático fraco, que extrai e focaliza na entrada do analisador de elétrons com energias menores que $2 \mathrm{meV}$, ejetados sobre todo o ângulo sólido de $4 \pi \mathrm{rad}$. Ele inclui um sistema de lentes de entrada, um Analisador Cilíndrico Dispersivo de $127^{\circ}$ (ACD-127 ${ }^{\circ}$ e um multiplicador de elétrons. O sistema de lentes consiste do estágio extrator e um conjunto de duas lentes eletrostáticas aceleradoras de três elementos cada, conectadas por uma região livre de campo, onde estão instalados dois conjuntos de placas defletoras. Alta resolução é obtida em função da queda muito rápida da eficiência de extração com o aumento da energia dos fotoelétrons. $\mathrm{O}$ analisador $\mathrm{ACD}-127^{\circ}$ elimina fotoelétrons energéticos emitidos dentro do ângulo sólido subentendido pelo eletrodo extrator. Todos os elementos do analisador são de molibdênio, cobertos por grafite aerossol, com objetivo de minimizar a emissão de elétrons secundários.

\section{RESULTADOS E DISCUSSÃO}

O espectrômetro foi testado utilizando luz sincrotrônica na linha de luz TGM do LNLS e um desempenho excelente foi obtido. A otimização, caracterização e calibração da escala de energia do espectrômetro foi realizada através dos estados iônicos bem conhecidos do Argônio ${ }^{2} \mathrm{P}_{3 / 2,1 / 2}$, localizados em 15,759 e 15,937 eV. Na Figura 3 é mostrado um espectro típico TPE do argônio cobrindo os estados iônicos ${ }^{2} \mathrm{P}_{3 / 2,1 / 2}$, com poder de resolução $(\mathrm{E} / \Delta \mathrm{E}) \mathrm{de}$ 716 , correspondendo a $17 \mathrm{meV}$ a $12 \mathrm{eV}$. O espectrômetro pode operar rotineiramente com este poder de resolução, que é o valor recorde já obtido nesta linha de luz. Considerando que nosso analisador $127^{\circ}$ possui um raio médio igual a $26 \mathrm{~mm}$ e tamanho das fendas de entrada e saída iguais a $1 \mathrm{~mm}$, seu poder de resolução operando na PES convencional é no máximo igual a $26^{11}$. Logo, o ganho em resolução do espectrômetro aplicando a Técnica de Campo Penetrante é maior que 27 vezes.

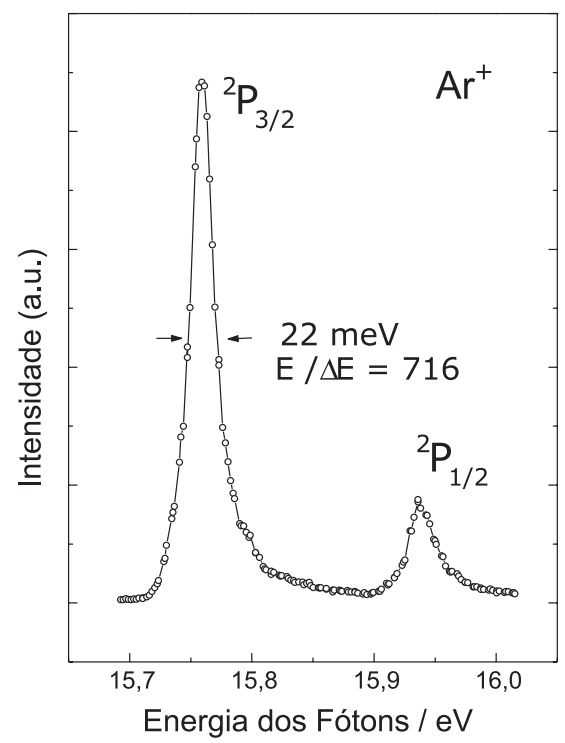

Figura 3. Espectro de Fotoelétrons de Limiares do argônio adquirido na linha de luz TGM do LNLS

A Figura 4 mostra a superposição de dois Espectros de Fotoelétrons de Limiares do argônio registrados em duas ocasiões diferentes, ilustrando a excelente estabilidade do aparelho. O segundo espectro foi registrado após a realização de uma série de medidas, envolvendo mudanças nas condições da linha de luz TGM, utilizando diferentes tamanhos de fendas de entrada e saída do monocromador e inserção e retirada de filtros metálicos de harmônicos superiores, de alumínio-magnésio-alumínio e ytérbio. Esta figura também mostra a ação destes filtros de harmônicos sobre a intensidade do feixe entregue na região de colisão, através dos espectros registrados utilizando exatamente as mesmas condições de operação do espectrômetro. Estes dados indicam a inviabilidade da aplicação dos filtros tanto de $\mathrm{Yb}$ como de $\mathrm{AlMgAl}$ na execução de espectroscopias de fotoelétrons altamente resolvidas, devido à alta queda na intensidade do fluxo de fótons. Na Espectroscopia de Fotoelétrons de Limiares a detecção é feita sobre $4 \pi$ sr e, portanto, em outras técnicas espectroscópicas sob condições de alta resolução angular e energética, a queda no sinal detectado será ainda maior que a registrada em nosso experimento.

$\mathrm{O}$ estudo da dependência da resolução e do fluxo de fótons do feixe de luz entregue na região de colisão com o tamanho das fendas utilizadas no monocromador da linha TGM está ilustrado na Figura 


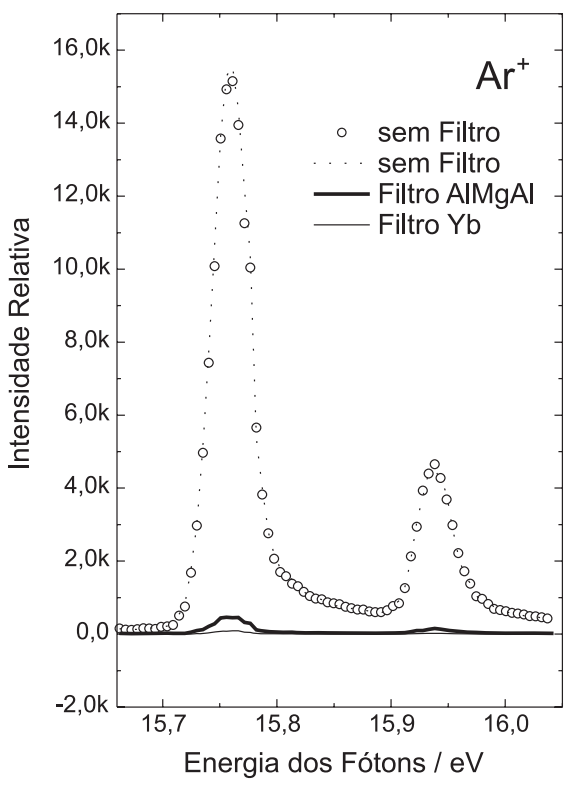

Figura 4. Ilustração da estabilidade do espectrômetro de Fotoelétrons de Limiares $e$ da ação dos filtros metálicos de harmônicos superiores de AlMgAl e de $\mathrm{Yb}$, sobre a intensidade do fluxo de fótons entregue na região de colisão com o alvo

5. Ao se aumentar o tamanho das fendas de entrada e saída de 100 para $250 \mu \mathrm{m}$ reduz-se a resolução por um fator de 1,2 e aumenta-se a intensidade do feixe de luz por um fator 5,7. Aumentando ainda mais o tamanho das fendas para $500 \mu \mathrm{m}$, a resolução cai por um fator de 2,1, enquanto a intensidade do feixe aumenta drasticamente por um fator de 13,3 . Isto evidencia que em alguns experimentos convém utilizar fendas maiores no monocromador, para se conseguir boa estatística nos dados adquiridos em um tempo menor, sem entretanto prejudicar consideravelmente a resolução espectral.

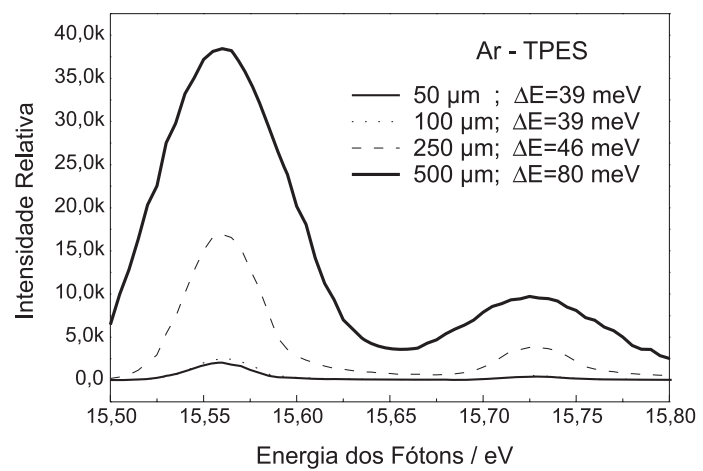

Figura 5. Comparação das intensidades do sinal detectado e resoluções energéticas registrados pela TPES em função do tamanho de fendas de entrada e saída do monocromador da linha TGM

As Figuras 6 e 7 mostram os Espectros de Fotoelétrons de Limiares do $\mathrm{O}_{2}$ e do $\mathrm{N}_{2}$ registrados, respectivamente, com fendas de entrada e saída no monocromador, de 300 e $100 \mu \mathrm{m}$. Estes espectros já foram anteriormente estudados por outros autores ${ }^{12-16} \mathrm{e} o$ nosso objetivo neste trabalho consistiu em explorar as possibilidades de estudos proporcionados pela TPES executada na linha de luz TGM do LNLS, que é uma linha até então reconhecida por fornecer baixa resolução energética. $\mathrm{O}$ espectro do $\mathrm{O}_{2}$ foi obtido em passos de $10 \mathrm{meV}$, cobrindo a região de energia de 12,0 a 22,0 eV e o do $\mathrm{N}_{2}$, com passos de $5 \mathrm{meV}$, na região de 15,2 a $18,0 \mathrm{eV}$. As principais bandas observadas nestes espectros concordam com aquelas registradas pela espectroscopia de fotoelétrons convencional.

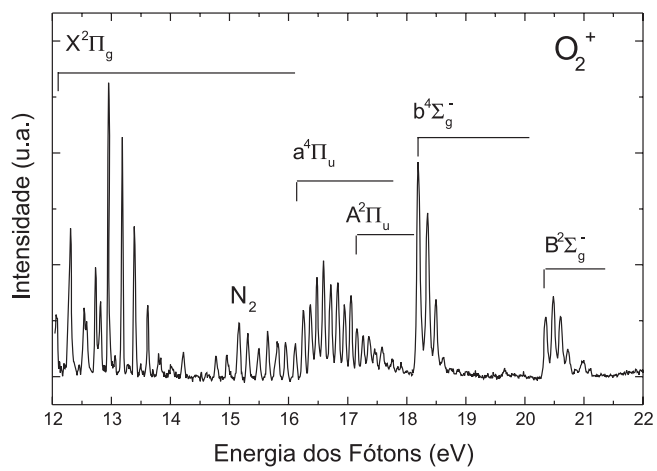

Figura 6. Espectro de Fotoelétrons de Limiares do $\mathrm{O}_{2}$ na faixa de energia de 12,0 a 22,0 eV, adquirido com passos de $10 \mathrm{meV}$ por canal. Picos adicionais foram registrados devido à contaminação da câmara de amostras com $\mathrm{N}_{2}$ e $\mathrm{H}_{2} \mathrm{O}$. A razão de contagem do pico mais intenso do estado $X^{2} \Pi_{g}$ foi de $11,0 \mathrm{kHz}$

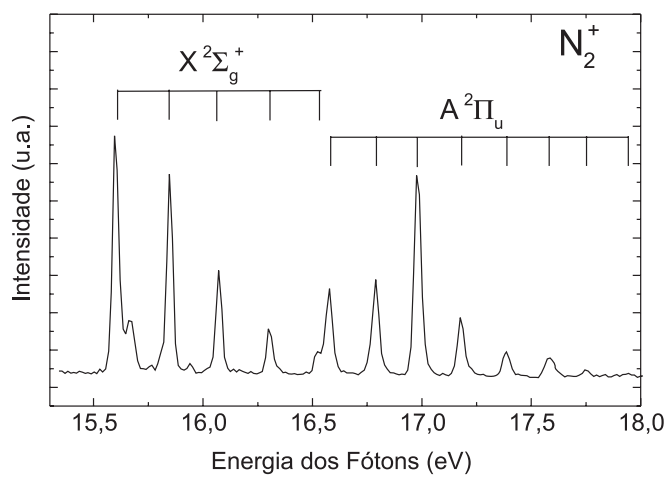

Figura 7. Espectro de Fotoelétrons Limiares do $\mathrm{N}_{2}$ sobre a faixa de energia de 15,2 a 18,0 eV, adquirido com passos de 5 meV por canal. A razão de contagem do pico correspondendo ao estado $v=0, \mathrm{X}^{2} \Sigma_{g}^{+}$foi de 7,0 $\mathrm{kHz}$. $O$ pequeno pico registrado logo acima deste estado deve-se a elétrons não limiares que não foram filtrados pelo analisador

Na região de 12 a 16,2 eV do espectro do $\mathrm{O}_{2}$ observa-se a formação de uma longa progressão vibracional associada aos níveis spinórbita do estado fundamental $\mathrm{X}^{2} \Pi_{\mathrm{g}}$ do íon $\mathrm{O}_{2}^{+}$, sobreposto a alguns picos devido à presença de água e $\mathrm{N}_{2}$ na câmara de alto vácuo. As intensidades vibracionais dos estados $\mathrm{a}^{4} \Pi_{u}$ e $\mathrm{A}^{2} \Pi_{u}$ do $\mathrm{O}_{2}{ }^{+}$são não Franck-Condon e, além disso, observam-se níveis vibracionais excitados, com altos números quânticos, que não são registrados pela PES. Atribui-se esta distorção a processos indiretos de ionização mais prováveis de ocorrer perto do limiar de ionização. No espectro TPE do $\mathrm{N}_{2}$ observam-se os níveis vibracionais $v=0$ a 4 do estado iônico $X^{2} \sum_{\mathrm{g}}^{+}$e também, $v=0$ a 7 do estado $\mathrm{A}^{2} \Pi_{\mathrm{u}}$. Devido às fendas menores utilizadas neste espectro, sua resolução é superior àquela do espectro do $\mathrm{O}_{2}$, observando-se totalmente resolvidas as componentes da progressão vibracional que aparecem nesta faixa de energia. O pequeno pico observado logo acima do pico $v=0, X^{2} \Sigma^{+}$ deve-se a elétrons não limiares que não foram filtrados pelo analisador. Os resultados obtidos nestes estudos evidenciam a eficácia do espectrômetro construído, demonstram que o poder de resolução da linha TGM é consideravelmente superior ao que se supunha até então, e encoraja o desenvolvimento de novos estudos espectroscópicos com alta resolução na linha de luz TGM do LNLS (Laboratório Nacional de Luz Síncrotron), de Campinas.

\section{CONCLUSÃO}

Construímos um Espectrômetro de Fotolétrons de Limiares que 
foi testado utilizando luz sincrotrônica na linha de luz TGM do LNLS e um excelente desempenho foi obtido. O espectrômetro opera em condições de alta estabilidade, com um poder de resolução de 716, correspondendo à resolução de 17 meV a 12 eV. Com este espectrômetro realizamos testes da ação de filtros metálicos de harmônicos superiores de $\mathrm{AlMgAl}$ e de $\mathrm{Yb}$ da luz na linha TGM, que revelaram a impossibilidade de execução de espectroscopias de fotoelétrons altamente resolvidas, devido à alta queda na intensidade do fluxo de fótons. Um estudo da dependência da resolução e do fluxo de fótons do feixe de luz entregue na região de colisão com o tamanho das fendas do monocromador da linha TGM evidenciou que a queda na resolução energética do feixe de luz não é drástica, à medida que se diminui o tamanho das fendas do monocromador. Entretanto, o sinal detectado na TPES pode cair por um fator de até 13,31. Nos espectros de Fotoelétrons de Limiares do $\mathrm{O}_{2}$ e do $\mathrm{N}_{2}$ foram registradas progressões vibracionais resolvidas, mostrando a importância de processos indiretos na fotoionização destas moléculas, e também demonstrando as possibilidades de estudos proporcionados pela TPES, executada na linha de luz TGM do LNLS.

\section{AGRADECIMENTOS}

Ao auxílio financeiro do Conselho Nacional de Desenvolvimento Científico e Tecnológico - CNPq, CAPES, FAPEMIG e LNLS.

\section{REFERÊNCIAS}

1. King, G. C.; Yencha, A. J.; Lopes, M. C. A.; J. Electron Spectrosc. Relat. Phenom. 2001, 114, 33.

2. Alagia, M.; Brunetti, B. G.; Candori, P.; Falcinelli, S.; Teixidor M. M.; Pirani, F.; Richter, R.; Stranges, S.; Vecchiocattivi, F.; J. Chem. Phys. 2004, $120,6980$.

3. King, G. C.; Avaldi, L.; J. Phys. B: At., Mol. Opt. Phys. 2000, 33, R215.

4. Cvejanovic, S.; Shiell, R. C.; Reddish, T. J.; J. Phys. B: At., Mol. Opt. Phys. 1995, 28, L707.

5. Avaldi, L.; Dawber, G.; Camilloni, R.; King, G. C.; Roper, M.; Siggel, M. R. F.; Stefani, G.; Zitnik, M.; Lisini, A.; Decleva, P.; Phys. Rev. A: At., Mol. Opt. Phys. 1995, 51, 5025.

6. Ellis, K.; Hall, R. I.; Avaldi, L.; Dawber, G.; McConkey, A.; Andric, L.; King, G. C.; J. Phys. B: At., Mol. Opt. Phys. 1994, 27, 3415.

7. Bolognesi, P.; Avaldi, L.; Lopes, M. C. A.; Dawber, G.; King, G. C.; MacDonald, M. A.; Villani, C.; Tarantelli, F.; Phys. Rev. A: At., Mol. Opt. Phys. 2001, 64, 2701.

8. Hall, R. I.; Avaldi, L.; Dawber, G.; Zubek, M.; Ellis, K.; King, G.C.; J. Phys. B: At., Mol. Opt. Phys. 1991, 24, 115.

9. Cvejanovic, S.; Read, F. H.; J. Phys. B: At., Mol. Opt. Phys. 1974, 7, 1180.

10. King, G. C.; Zubek, M.; Rutter, P. M.; Read, F. R.; J. Phys. E 1987, 20, 440 .

11. Moore, J. H.; Davis, C. C.; Coplan, M. A.; Building Scientific Apparatus, Addison-Wesley Publishing Company Inc: New York, 1989.

12. Zubek, M.; King, G. C.; Rutter, P. M.; J. Phys. B: At., Mol. Opt. Phys. 1988, 21, 3585 .

13. Dehmer, P. M.; Miller P. J.; Chupka, W. A.; J. Chem. Phys. 1984, 80, 1030.

14. Tabche-Fouhaile, A.; Ito, K.; Nenner, I.; Frölich, H.; Guyon, P. M.; J. Chem. Phys. 1982, 77, 182.

15. Ellis, K.; Hall, R. I.; Avaldi, L.; Dawber, G.; McConkey, A.; Andrié, L.; King, G. C.; J. Phys. B: At., Mol. Opt. Phys. 1994, 27, 3415.

16. Baltzer, P.; Wannberb, B.; Karlsson, L.; Carlsson-Göthe, M; Phys. Rev. A: At., Mol., Opt. Phys. 1992, 45, 4374. 